Urologe $2016 \cdot 55: 245$

DOI 10.1007/s00120-016-0033-5

Online publiziert: 28. Januar 2016

๑) Springer-Verlag Berlin Heidelberg 2016

CrossMark

\title{
Lucas Hirner
}

Klinik für Urologie, Kinderurologie und Uroonkologie, Universitätsklinikum Essen (AöR), Essen, Deutschland

\section{Erste Geburt nach erfolgreicher Uterustransplantation}

\author{
Originalpublikation \\ Brännström $M$, Johannesson $L$, Bokström $H$, \\ Kvarnström N, Mölne J, Dahm-Kähler P, \\ Enskog A, Milenkovic M, Ekberg J, Diaz- \\ Garcia C, Gäbel M, Hanafy A, Hagberg H, \\ Olausson M, Nilsson L (2015) Livebirth after \\ uterus transplantation. Lancet \\ 385(9968):607-616
}

Die Publikation beschreibt die erste Geburt eines gesunden Kindes nach Uterustransplantation bei einer 35-jährigen Patientin.

Die Patientin ist an einem Mayer-Rokitansky-Küster-Hauser-Syndrom (angeborene Fehlbildung des weiblichen Genitals durch Hemmungsfehlbildung der Müller-Gänge; Ovarialfunktion ist nicht gestört) erkrankt. Bei ihr sind Uterus und eine Niere nicht angelegt. Das Spenderorgan war ein Uterus einer postmenopausalen, mit der Patientin gut befreundeten 61-jährigen Spenderin (para 2).

43 Tage nach der Transplantation setzte der erste Zyklus ein (durchschnittliche Zykluslänge: 32 Tage). Die Patientin erhielt eine immunsuppressive Dreifachtherapie (Tacrolimus, Azathioprin und Glukokortikoide). Nach einer In-vitroFertilisation und Kryokonservierung von 11 Embryonen vor der Transplantation erhielt die transplantierte Patientin 1 Jahr nach dem ersten Zyklus einen Embryo transferiert, was in einer Schwangerschaft resultierte. Während der Schwangerschaft waren die Entwicklungsparameter des Kindes (Femurlänge, Gewicht) jederzeit normal. Es kam zu einer milden Abstoßungsreaktion. Aufgrund einer Präeklampsie erfolgte nach 31 Wochen und 5 Tagen eine Hospitalisierung. Dort wurde nach Detektion einer abnormalen Kardiotokographie ein Kaiserschnitt durchgeführt. Ein gesundes, dem Gestationsalter entsprechendes normalgewichtiges Kind (1775 g) wurde geboren (Apgar-Score 9, 9, 10). Die histologische Untersuchung der Plazenta war unauffällig. Die Patientin konnte 3 Tage nach der Operation, das Neugeborene 16 Tage nach Geburt aus dem Krankenhaus entlassen werden. Die Patientin wurde darüber informiert, dass aufgrund der Nebenwirkungen der Immunsuppressiva der Uterus spätestens nach einer zweiten Schwangerschaft explantiert werden sollte. Die Uterustransplantation ist eine mögliche Therapieoption bei uteriner Ursache einer Unfruchtbarkeit.

\section{Korrespondenzadresse}

\section{Hirner}

Klinik für Urologie, Kinderurologie und Uroonkologie, Universitätsklinikum Essen (AöR) Hufelandstraße 55, 45122 Essen, Deutschland lucas.hirner@uk-essen.de

Interessenkonflikt. L. Hirner gibt an, dass kein Interessenkonflikt besteht. 